\title{
MONOGRAFÍA
}

\section{INSUFICIENCIA CARDÍACA Y DIABETES}

\section{HEART FAILURE AND DIABETES}

\author{
María Carolina Panzitta ${ }^{1}$ Isaac Sinay ${ }^{2}$
}

\section{RESUMEN}

Cuando nos enfrentamos a la diabetes mellitus (DM) y a la insuficiencia cardíaca (IC) nos encontramos con dos patologías que presentan aspectos en común. Uno de ellos es la alta prevalencia que existe en la población general. A su vez, la DM se postula como un factor de riesgo independiente para el desarroIlo de IC, no sólo por los efectos de la hiperglucemia sostenida sino por el conjunto de comorbilidades asociadas que elevan el riesgo de estos pacientes de desarrollar cardiopatía isquémica y fallo cardíaco.

El objetivo del presente estudio es realizar una revisión de la diabetes y la hiperglucemia como un fenómeno que promueve el desarrollo de insuficiencia cardíaca.

Su abordaje diagnóstico incluye una serie de estudios complementarios que sumados a un correcto examen clínico permiten una detección precoz y tratamiento oportuno.

Los inhibidores de la enzima convertidora de angiotensina, los betabloqueantes y los antagonistas de los receptores de mineralocorticoides continúan siendo la piedra angular para un correcto manejo de la IC sumado al control de la glucemia y las enfermedades concomitantes. En este aspecto se destacan la empaglifozina y el liraglutide como fármacos que favorecen el descenso del riesgo cardiovascular, así como el uso de las estatinas. Pero su abordaje terapéutico comprende no sólo el tratamiento de la IC instalada y sus complicaciones sino la prevención de la diabetes y su screening precoz.

Palabras clave: obesidad, diabetes mellitus, insuficiencia cardíaca.

Revista de la Sociedad Argentina de Diabetes 2018; Vol. 52 (25-34)

\section{ABSTRACT}

When we face diabetes mellitus and heart failure (HF), we find two pathologies that have aspects in common. One of them is the high prevalence that exists in the general population. In turn, diabetes is presented as an independent risk factor for the development of HF, not only due to the effects of sustained hyperglycemia, but it is the set of associated comorbidities that increase the risk of these patients to develop ischemic heart disease and heart failure.

The objective of the present study is to conduct a review of diabetes and hyperglycemia as a phenomenon that promotes the development of heart failure.

Its diagnostic approach includes a series of complementary studies that, together with a correct clinical examination, allow early detection and timely treatment.

Angiotensin-converting enzyme inhibitors (ACEIS), beta-blockers (BBs) and mineralocorticoid receptor antagonists (MRAs) continue to be the cornerstone for the correct management of $\mathrm{Cl}$, in addition to the control of glycemia and concomitant diseases. Empaglifozin and liraglutide stand out in this aspect, as drugs that favor the decrease in cardiovascular risk, as well as the use of statins.

But its therapeutic approach includes not only the treatment of the installed HF and its complications but also the prevention of diabetes and the early screening of this condition.

Key words: obesity, diabetes mellitus, heart failure.

Revista de la Sociedad Argentina de Diabetes 2018; Vol. 52 (25-34)
1 Médica especialista en Clínica Medica, especializada en Diabetes, Hospital Municipal Pigüe, Provincia de Buenos Aires, Argentina

2 Médico Endocrinólogo, asesor de la Unidad Diabetes del Instituto Cardiovascular de Buenos Aires, Ciudad Autónoma de Buenos Aires, Argentina

Contacto de la autora: María Carolina Panzitta

E-mail: susana.carolinapanzitta85@gmail.com
Correspondencia: Buenos Aires 73 (B8170CXA), Pigüe, Provincia de Buenos Aires, Argentina

Tel.: (0291) 154438236

Fecha de trabajo recibido: 09/04/18

Fecha de trabajo aceptado: 17/04/2018

Conflictos de interés: Isaac Sinay declara que recibió honorarios por asesorías, docencia o actividad en estudios clínicos de Sanofi Aventis, Servier, Merck Sharp \& Dohme, AstraZeneca, Boehringer Ingelheim y Janssen.

\author{
ABREVIATURAS \\ AGL: ácidos grasos libres. \\ ANG: angiotensina. \\ ARM: antagonistas de los receptores de mineralocorticoides. \\ BB: betabloqueantes. \\ DM: diabetes mellitus. \\ FE: fracción de eyección. \\ HTA: hipertensión arterial. \\ HVI: hipertrofia del ventrículo izquierdo. \\ IC: insuficiencia cardíaca.
}

IECA: inhibidores de la enzima convertidora de angiotensina. IRS: sustrato del receptor de insulina.

ON: óxido nítrico.

PPAR: receptores activados por proliferadores de peroxisomas. SF: sulfonilureas.

SRA: sistema renina angiotensina.

TAS: tensión arterial sistólica.

TGF-31: factor de crecimiento transformante beta 1.

VEGF: factor de crecimiento vascular endotelial. 


\section{INTRODUCCIÓN}

La diabetes se ha convertido en una epidemia mundial ${ }^{1}$ y constituye una de las principales causas de muerte prematura, especialmente por el aumento del riesgo de enfermedad cardiovascular que conlleva, reportándose una mortalidad aproximada del $50 \%{ }^{2}$.

La prevalencia de insuficiencia cardíaca en personas con diabetes varía según la literatura entre un 12 y un 30\%3,4,5. Tal como lo describió The Framingham Heart Study, la diabetes es un factor de riesgo independiente para el desarrollo de enfermedad cardiovascular, con un aumento del riesgo entre dos a cinco veces de padecer insuficiencia cardíaca.

El objetivo del presente estudio es realizar una revisión de la diabetes y la hiperglucemia como un fenómeno que promueve el desarrollo de insuficiencia cardíaca.

\section{Fisiopatología de la insuficiencia cardíaca en la diabetes mellitus}

La fisiopatología de la insuficiencia cardíaca en la diabetes responde a múltiples causas, donde no sólo la hiperglucemia impacta en forma directa sobre el miocardio sino que es la interacción entre múltiples comorbilidades de la diabetes lo que potencia este daño y lleva finalmente a falla cardíaca. Entre ellos se destacan los siguientes factores:

a) Hiperactivación del eje SRA e insulinorresistencia ${ }^{6,78,9}$

Tanto la ANG como la aldosterona inducen la fosforilación en serina del IRS por diferentes mecanismos en múltiples tejidos y genera insulinorresistencia. Por otra parte presentan un rol activo en la generación y estimulación de especies reactivas del oxígeno, lo cual favorece el daño celular y la apoptosis (tanto a nivel cardíaco como de la célula beta).

Este proceso genera un círculo vicioso donde la hiperinsulinemia del paciente con DM2 estimula la activación del eje SRA, el cual dentro de sus efectos moleculares deletéreos incrementaría la insulinorresistencia ${ }^{10}$. A esto se suma la regulación anormal de este sistema en pacientes con obesidad visceral, quienes presentan hiperactivación ya sea por falta de inhibición por parte de los péptidos antagonistas o bien por hiperactividad del sistema simpático estimulado fundamentalmente por la hiperinsulinemia crónica presente en estos individuos ${ }^{5,11}$.

b) Impacto a nivel cardíaco $^{12}$
La Ag II (en sinergia con la aldosterona) actuaría sobre el cardiomiocito al aumentar vía insulinorresistencia y vía MAPK (con activación de la vía de las caspasas y del TGF-B1) la apoptosis y la mayor producción de colágeno por los fibroblastos cardíacos. Todo esto genera efectos profibróticos que favorecen el remodelado cardíaco, la HVI y el aumento de rigidez, todos procesos críticos para generar insuficiencia cardíaca. Por ello al pensar en SRA su impacto en coronariopatía y remodelado hace que su hiperactividad sea un fuerte predictor de falla cardíaca.

\section{c) Hipertensión arterial}

La hipertensión se ha identificado como un factor de riesgo para el desarrollo de enfermedad cardiovascular así como de diabetes, con un aumento entre dos a tres veces el riesgo si se compara con personas con tensión arterial normal.

A nivel del árbol vascular acelera el proceso de arterioesclerosis, favorece un aumento del "shear stress" en la pared vascular ${ }^{14}$ con aumento compensador de su diámetro y conduciría a largo plazo a un incremento en la rigidez y disminución de la compliance, tanto de grandes arterias como a nivel de la microvasculatura. El denominador común de estos eventos es el desarrollo de HVI y aumento de la resistencia periférica.

A nivel cardíaco ${ }^{13,14,15,16}$ a través de estímulos de crecimiento, hemodinámicos y neurohumorales favorece el desarrollo de HVI al comprometer la reserva vasodilatadora de la circulación coronaria e incrementar la demanda de oxígeno por el miocardio. A largo plazo conduce a dilatación de las cavidades y pérdida de la función contráctil. En etapas tempranas el deterioro del llenado es causado predominantemente por una disminución de la relajación ventricular durante la diástole temprana, estadio en el cual los pacientes pueden presentar una capacidad reducida al ejercicio como única manifestación clínica. Un progresivo descenso en la función del VI conlleva al desarrollo de IC. El riesgo aumenta un 55\% por cada incremento de la TAS de $20 \mathrm{mHG}$.

d) Microvasculopatía

El control de la microcirculación puede dividirse en: un control central, determinado por la acción del sistema nervioso autónomo, y un control local, donde intervienen fenómenos a corto plazo (autorregulación) y a largo plazo, destacando el fenómeno de angiogenia ${ }^{17}$.

A nivel de la microcirculación coronaria ${ }^{18}$, debido 
a la actividad metabólica del miocardio, se produce una significativa extracción de oxígeno de la sangre arterial, con importante desaturación a nivel del seno coronario. Este hecho implica la necesidad de generar aumentos del flujo ante cualquier adaptación que incremente la actividad metabólica.

La diabetes afecta todos los mecanismos de regulación de la microvasculatura ${ }^{19,20}$. En primer lugar el daño que genera la hiperglucemia sobre los nervios periféricos, y la consecuente neuropatía autonómica cardíaca conduce a una alteración de la respuesta vascular coronaria a la estimulación simpática, con disminución de la vasodilatación. Esto determina que ante un estímulo de estrés o aumento de la demanda metabólica la vasculatura del paciente con DM responderá con una tasa de flujo cardíaco menor. Sumado a esto, la neuropatía conduce a una mayor tasa de infarto silente y se asocia a una disminución de la función diastólica ${ }^{21,22}$.

A su vez a nivel local la hiperglucemia crónica genera aumento del grosor de la membrana basal capilar y mayor rigidez vascular, con una menor perfusión coronaria en diástole y, por lo tanto, mayor riesgo de isquemia especialmente a nivel del subendocardio. El segundo efecto es la disfunción endotelial, con aumento en la producción de sustancias vasoconstrictoras y disminución de la producción de ON lo cual favorece un estado de vasoconstricción persistente que se sumará a la hiperactivación simpática y del SRA. Un tercer efecto es la reducción en pacientes diabéticos del flujo coronario de reserva normal y de la angiogénesis en respuesta a la hipoxia. Esto último se explica por un descenso en la expresión del VEGF.

Por lo tanto, la hiperglucemia crónica sumada a la insulinorresistencia periférica determinan un corazón vulnerable a la isquemia, con incapacidad de aumentar la tasa de perfusión coronaria ni su densidad angiogénica en presencia de hipoxia, a lo cual se le suma la disfunción endotelial característica, el aumento del estrés oxidativo y los fenómenos de glicación y fibrosis intersticial. Todo esto conducirá a largo plazo a disminución de la compliance ventricular y disfunción del VI con la consecuente falla cardíaca.

e) Aterosclerosis y enfermedad coronaria $23,24,25,26$

El $75 \%$ de los pacientes con DM2 muere por enfermedad cardiovascular. Dos tercios de estas muertes son manifestación de enfermedad coronaria isquémica la cual, a su vez, es la causa subyacente de la mayoría de los pacientes con disfunción sistólica del VI.
Su desarrollo, así como la susceptibilidad de los pacientes con diabetes a la isquemia, no sólo resulta de la hiperglucemia crónica, sino de la insulinorresistencia, dislipemia, hipercoagulabilidad, alteración de la secreción de sustancias locales y menor respuesta a la injuria, con la formación de placas de ateromas más vulnerables a la ruptura. Debido a este mecanismo multicausal, al momento del tratamiento debe realizarse un abordaje de múltiples factores de riesgo para evitar el desenlace común de estos pacientes, que es la isquemia y la falla cardíaca.

\section{f) Insulinorresistencia y síndrome metabólico}

La insulinorresistencia, definida como la disminución en la capacidad de la insulina de ejercer sus efectos metabólicos en los tejidos periféricos, responde a mecanismos genéticos, epigenéticos y ambientales. Entre ellos se destacan: la alteración en la señalización intracelular con resistencia postreceptor, glucolipotoxicidad, sumado al rol del adipocito visceral como órgano endocrino, con producción de adipocitokinas ${ }^{27,28,29,30}$.

Dentro de sus efectos deletéreos se mencionan, en primer lugar, el desarrollo de diabetes, con disfunción de la célula beta, el desarrollo de HTA, explicado tanto por el desarrollo de disfunción endotelial, tendencia a la vasoconstricción y la hiperactividad simpática en pacientes obesos, con un aumento de la resistencia periférica y de la reabsorción de sodio y agua por el túbulo renal, con activación del SRA. A esto se suma la dislipemia característica de estos pacientes, estado procoagulante y proinflamatorio, y el efecto de las adipocitokinas. Todos estos cambios constituyen el síndrome metabólico, con un alto impacto a nivel cardiovascular, destacando a la macrovasculopatía como un evento temprano en el contexto de insulinorresistencia, con alta relación con la tolerancia alterada a la glucosa ${ }^{31,32,33}$.

Enfocandonos en el corazón ${ }^{34}$, la insulinorresistencia es responsable de múltiples alteraciones a nivel estructural y funcional. En particular, anormalidades en las proteínas de contracción y disminución de los fenómenos de relajación, injuria directa de cardiomiocitos, fenómenos de fibrosis por glicación del intersticio, alteración de la microvasculatura y disminución de la reserva coronaria, hiperactivación de los sistemas neurohormonales y cambios en la utilización de substratos.

Respecto de este último punto, normalmente a nivel cardíaco ${ }^{35,36}$ el aumento de la insulinorre- 
sistencia reduce la captación de glucosa por los cardiomiocitos que pasa a emplear más AGL con peor eficiencia metabólica y mayor proclividad a la miocardiopatía como se comentará a continuación.

\section{g) Miocardiopatía diabética}

El término miocardiopatía diabética estrictamente se refiere a la rara circunstancia de disfunción ventricular en pacientes con DM en ausencia de enfermedad coronaria e hipertensión. A continuación se describen algunos de los mecanismos moleculares que conducen a la misma, varios basados en estudios en animales 37,38 .

En primer lugar se describe un metabolismo de los $\mathrm{AGL}^{39,40}$ alterado en el cardiomiocito, recordando que en condiciones normales del 70 al $80 \%$ del consumo de energía miocárdica proviene de la oxidación de los ácidos grasos libres y sólo el resto depende de la glucólisis (el cual se vuelve fundamental ante un aumento de las demandas metabólicas).

En el paciente con diabetes existe un aumento en la lipólisis y en la producción hepática de triglicéridos, lo cual determinará una oferta excesiva de AGL al corazón, cuya entrada está incrementada por aumento de la expresión de CD36. Esto determina una mayor utilización de lípidos y menor oxidación de glucosa.

Los ácidos grasos libres estimulan la expresión de receptores PPAR alfa y ello genera aumento de la beta oxidación mitocondrial que, sin embargo, no llega a compensar el aumento en su captación y una disminución de la acción de la piruvato deshidrogenasa y la utilización de glucosa. Todo esto conduce a una acumulación de lípidos intracelulares, lipotoxicidad (con aumento en la formación de ceramidas), disfunción mitocondrial y aumento del estrés oxidativo, todos mecanismos involucrados en el desarrollo y progresión de miocardiopatía e insuficiencia cardíaca.

En segundo lugar se describe una situación de vulnerabilidad a la injuria miocárdica, ante situaciones de isquemia o bien ante una insuficiencia cardíaca descompensada. La presencia de insulinorresistencia suprime el mecanismo de adaptación del miocardio ante la hipoxia. A esto se agrega la falla del preacondicionamiento isquémico, lo cual conduce a mayor apoptosis del cardiomiocito y peor pronóstico.

En tercer lugar existiría una alteración en el acoplamiento contracción-excitación por una disminución en el eflujo del calcio que conduciría a prolongación del potencial de acción y disfunción diastólica y sistólica.
Por último se describe una alteración del intersticio donde el aumento en la formación de productos de la glicosilación avanzada y su depósito en proteínas del colágeno conducen a fibrosis del intersticio y de las paredes arteriales, con la consecuente rigidez miocárdica, alteración de la relajación y posterior falla diastólica. Esto se potencia por la acción de la angiotensina vía AT1 y aumento del estrés oxidativo vía RAGE. La combinación de los factores anteriormente citados conduce a la remodelación del miocardio, con disminución de la función sistólica y consecuente retroalimentación positiva del SRA y del sistema nervioso autónomo. La estimulación de estos sistemas neurohormonales distorsiona la estructura normal del ventrículo izquierdo (hipertrofia y dilatación) que en caso de no controlarse conducirá a falla de bomba, arritmias y muerte.

\section{Clasificación de la \\ insuficiencia cardíaca ${ }^{41,42,43,44,45}$}

El síndrome de IC se define como la incapacidad de mantener adecuadamente la circulación acorde con los requerimientos metabólicos en reposo y en esfuerzo del organismo a pesar de condiciones de llenado adecuadas o hacerlo pero con elevación de las presiones de llenado.

Puede clasificarse según su evolución en aguda o crónica, acorde a la fracción de eyección, según la gravedad de los síntomas (clasificación funcional de la New York Heart Association) o bien según cambios estructurales y síntomas (ACCFF/AHA). De ahora en adelante nos enfocaremos en la IC crónica.

\section{Insuficiencia cardíaca crónica}

\section{a) Clínica}

Al abordar un paciente con IC, la herramienta fundamental que permitirá llegar a un adecuado diagnóstico aún es una anamnesis detallada y un examen físico completo.

- Síntomas: incluyen aquellos debidos a retención hidrosalina, tales como disnea, ortopnea y disnea paroxística nocturna. Cabe mencionar la disnea como síntoma no sólo de IC sino también como manifestación de un síndrome coronario agudo con alta tasa de presentación subclínica en la población con diabetes ${ }^{46}$.

La debilidad, fatiga e intolerancia al ejercicio y frialdad de miembros corresponderían a síntomas de bajo gasto cardíaco, que pueden evolucionar en casos graves a confusión mental y shock. 
- Signos: mediante el examen físico se constatarán signos de sobrecarga de volumen y de presión, agrandamiento ventricular, hipertensión pulmonar, presiones de llenado elevadas y disminución del gasto cardíaco.

En líneas generales son cuatro los signos que indican progresión y marcan la severidad del cuadro: hipotensión, taquicardia, vasoconstricción periférica con palidez y cianosis, y amplitud de pulso disminuida o pulso paradójico, patognomónico de falla ventricular izquierda severa.

El desplazamiento del latido apical es el signo más sensible y específico en la insuficiencia cardíaca con función sistólica disminuida. Otros signos con alto valor predictivo son el ritmo de galope, la ingurgitación yugular y el reflujo hepatoyugular.

\section{b) Exámenes complementarios}

Para un adecuado abordaje diagnóstico deberá solicitarse una serie de estudios de laboratorio, destacando en la población con diabetes que la presencia de albuminuria así como de hemoglobina glicosilada aumentada se presentan como fuertes predictores de desarrollo de IC $\mathrm{C}^{47,48}$.

Dentro de los exámenes de rutina se destaca el electrocardiograma (ECG), estudio con alta sensibilidad y bajo costo ${ }^{49}$. Existe cierta controversia respecto del valor de los programas de cribado para detectar la enfermedad arterial coronaria subclínica en pacientes con DM mediante pruebas no invasivas. En el estudio Detection of Silent Myocardial Ischemia in Asymptomatic Diabetic Subjects (DIAD) ${ }^{50}$, diseñado para determinar la efectividad del screening de cardiopatía isquémica en pacientes diabéticos tipo 2, su principal conclusión fue que la prueba de isquemia no sirvió para modificar la evolución clínica ni el pronóstico de los pacientes.

Las indicaciones al respecto de la Sociedad Americana de Diabetes 2018 son: evaluar presencia de cardiopatía isquémica en aquellos pacientes con síntomas cardíacos atípicos, clínica sugestiva de enfermedad vascular periférica y/o paciente con electrocardiograma en reposo anormal, existiendo controversia acerca del screening en pacientes asintomáticos, sin tener indicación precisa hasta el momento ${ }^{51,52}$.

Como segundo estudio fundamental en este contexto se destaca el ecocardiograma doppler ${ }^{53,54}$, sin dudas, la prueba diagnóstica de mayor valor aislado en pacientes con IC. Su utilización debe ser de rutina en la evaluación inicial y en el seguimiento.
Por último se menciona el péptido natriurético tipo B (BNP) y la fracción aminoterminal del propéptido natriurético tipo $B$ (NT-proBNP), los cuales se utilizan como examen inicial para el diagnóstico de IC, especialmente cuando no está disponible el ecocardiograma. Valores por encima del punto de corte exigen mayor evaluación, mientras que valores normales excluyen el diagnóstico de IC y denotan un alto valor predictivo negativo y su utilidad en casos de dudas diagnósticas.

Otros estudios diagnósticos tales como ergometría, ecocardiograma de esfuerzo, RMN, tomografía axial multicorte o bien estudios invasivos se solicitarán según la indicación pertinente, escapando su detalle el objetivo de esta revisión.

\section{c) Tratamiento}

Los objetivos del tratamiento de la IC crónica son reducir la mortalidad y los síntomas, aumentar la capacidad de ejercicio, disminuir las internaciones, proveer una mejor calidad de vida y prevenir los eventos isquémico-necróticos, remodelación y apoptosis.

- Medidas generales: dentro de las medidas no farmacológicas se describen el cuidado del peso, restringir la ingesta de sodio y la de líquidos en aquellos con síntomas graves o bien con hiponatremia, el consumo moderado de alcohol y el abandono del habito tabáquico. Los pacientes con IC deberán recibir la vacuna antineumocócica y antigripal. Prescribir actividad física en aquellos pacientes estables, sin contraindicaciones para evitar atrofia muscular y mejorar la tolerancia al esfuerzo.

- Tratamiento farmacológico

- IC con FE disminuida

Los tres antagonistas neurohormonales, IECA o ARA, betabloqueantes y antagonistas de los receptores de mineralocorticoides constituyen los tres principales agentes en el tratamiento de la IC sistólica.

. Inhibidores de la enzima convertidora de angiotensina

Han mostrado reducción de la morbilidad y mortalidad tanto en pacientes con IC con FE reducida así como en pacientes asintomáticos con disfunción sistólica del VI en los cuales reduciría el desarrollo de IC, hospitalización y muerte, tal como lo describe el estudio Studies Of Left Ventricular Dysfunction (SOLVD), teniendo igual impacto en pacientes con y sin DM como se analiza en el Studies of Left Ventricular Dysfunction Trials and Registry (RESOLVD) ${ }^{55}$.

Betabloqueantes 
Reducen la morbilidad y mortalidad en pacientes sintomáticos, más allá del tratamiento con IECA, existiendo consenso con el uso complementario de ambos fármacos, especialmente en aquellos que presenten como antecedentes asociados un infarto de miocardio. Se reportan beneficios similares en pacientes con y sin $\mathrm{DM}^{56}$ a pesar que en esta subpoblación se describen algunos efectos adversos como disminución de la insulinosensibilidad o hipoglucemia, que no limitan su uso ya que los efectos beneficiosos sobrepasan los posibles efectos adversos.

Antagonistas de los receptores de mineralocorticoides

Recomendados por demostrar reducir la tasa de hospitalización y mortalidad en todos los pacientes con $\mathrm{FE} \leq 35 \%$, con síntomas persistentes (NYHA) clase II-IV, a pesar del tratamiento con IECA (o ARA) y BB.

. Antagonistas de los receptores de angiotensina y neprilisina

Su acción se basa en la inhibición conjunta del SRA y el sistema de endopeptidasas al lograr una menor degradación de ciertas sustancias (tales como el péptido natriurético atrial, bradicinina, entre otras) con efectos beneficiosos a nivel cardiovascular. El estudio Prospective Comparison of ARNI with ACEI to Determine Impact on Global Mortality and Morbidity in Heart Failure (PARADIGM HF) ${ }^{57}$, el cual compara sacubitril/valsartán con enalapril, mostró disminución de la mortalidad por todas las causas, cardiovascular y menor tasa de hospitalización, y detectó iguales resultados cuando se analizó el subgrupo de pacientes con tolerancia alterada a la glucosa y diabetes y aquellos con diagnóstico desconocido ${ }^{58}$.

Actualmente sólo está indicado en reemplazo del IECA para reducir el riesgo de hospitalización por IC y muerte en pacientes sintomáticos a pesar de tratamiento óptimo.

Antagonistas de los receptores tipo 1 de angiotensina

Recomendados como alternativa en aquellos pacientes que no toleran Ios IECA.

Otros fármacos como diuréticos, ivabradina o isosorbide dinitrato/hidralazina presentan recomendaciones específicas, sin evidencia clara de reducción de la mortalidad con su uso. Cabe considerar el empleo de digoxina en pacientes sintomáticos a pesar del tratamiento óptimo y completo con IECA, BB y ARM para reducir el riesgo de hospitalización y el uso de anticoagulantes orales en aquellos con IC y fibrilación auricular, trombo intracavitario por imágenes o embolia sistémica.

Por último contamos, dentro del tratamiento no farmacológico, con la terapia de resincronización cardíaca, el cardiodesfibrilador implantable y el trasplante cardíaco, terapias reservadas en pacientes con IC avanzada o indicaciones específicas.

\section{IC con FE conservada-intermedia}

No existe clara evidencia respecto de una reducción de la mortalidad con alguna terapia en particular, siendo el objetivo primordial en estos pacientes realizar un tratamiento efectivo para aliviar los síntomas y tratar las comorbilidades asociadas. Es recomendable evitar elevaciones abruptas de la tensión arterial, isquemia y fibrilación auricular al igual que una excesiva reducción de la precarga. Los únicos fármacos recomendados son los diuréticos con el objetivo de conseguir una mejoría sintomática.

\section{Manejo de la glucemia en pacientes con IC $^{59}$}

En estos pacientes el objetivo de control glucémico debe pactarse teniendo en cuenta el contexto clínico, el riesgo de descompensación y hospitalización, y la presencia de posibles hipoglucemias $^{60}$. Existe controversia respecto del valor de corte de $\mathrm{HbA} 1 \mathrm{c}$, y se describe una relación, tipo curva en J, cuando se menciona su asociación con mortalidad por todas las causas, donde tanto valores muy elevados como muy bajos serian perjudiciales ${ }^{61}$, lo cual concuerda con estudios como el Action to Control Cardiovascular Risk in Diabetes (ACCORD).

- Metformina

Recomendada como la droga de primera elección en pacientes con DM e IC compensada, con estudios que reportan disminución de la tasa de mortalidad y hospitalización con escasos efectos adversos. Respecto de este último punto, existen numerosos estudios que analizaron el riesgo de desarrollar acidosis láctica, sin existir evidencia de calidad al respecto ${ }^{62}$, como lo demuestra la revisión de Cochrane ${ }^{63}$, la cual no detectó diferencias en cuanto a lactacidemia ni casos de acidosis láctica entre 347 estudios analizados que incluían más de 3.000 pacientes. En un estudio de casos y controles que incluía pacientes con diagnóstico reciente de DM e IC, los cuales fueron expuestos a antidiabéticos orales vs placebo, el uso de metformina (OR ajustado $0,65,0,48-0,87$ ) o metformi- 
na con o sin otros agentes (OR 0,72, 0,59-0,90) se asoció a disminución de la mortalidad, mientras que con otros antidiabéticos orales o con insulina el efecto fue neutro ${ }^{64}$. Sin embargo su uso está contraindicado en aquellos pacientes con insuficiencia renal y/o hepática severa, o bien en casos de IC descompensada.

- Sulfonilureas y meglitinidas

Existe controversia respecto del uso de sulfonilureas por cierta evidencia que indica un empeoramiento del curso clínico de la IC, basado mayoritariamente en estudios observacionales. Los resultados de ensayos clínicos controlados como The UK Prospective Diabetes Study (UKPDS), Action in Diabetes and Vascular Disease (ADVANCE) y ACCORD no mostraron un aumento del riesgo cardiovascular en cuanto a morbimortalidad en pacientes tratados con SF. El estudio en marcha Cardiovascular Outcome Study of Linagliptin Versus Glimepiride in Patients with Type 2 Diabetes (CAROLINA), que finalizará en 2019 y que compara linagliptina vs glimepirida en pacientes con DM, ayudará a dilucidar la seguridad cardiovascular de estas drogas. Mientras tanto, y dado que la glibenclamida se relaciona con disminución del pre-acondicionamiento isquémico con mayor tasa de hipoglucemias y aumento del riesgo cardiovascular, se recomienda no utilizarla de primera elección. Respecto de las meglitinidas, por su diferente farmacocinética y su corta duración de acción, no presentan asociación con riesgo cardiovascular.

- Tiazolidinedionas (glitazonas)

Estos fármacos inducen retención hidrosalina y expansión de volumen lo cual puede desencadenar o empeorar el cuadro de un paciente con IC, por lo que no están recomendados como fármacos de elección. Sin embargo en el estudio Prospective Pioglitazone Clinical Trial in Macrovascular Events (PRO active) ${ }^{65}$ el uso de pioglitazona se asoció a un aumento de la incidencia de IC pero sin aumento de la mortalidad demostrando a su vez mejoría de algunos parámetros de riesgo $\mathrm{CV}$.

- Inhibidores de DPP-4 y análogos del GLP-1

Existe escasa experiencia respecto del uso de estas drogas en pacientes con IC establecida. Tres recientes estudios clínicos (Saxagliptin and Cardiovascular Outcomes in Patients with Type 2 Diabetes Mellitus-Thrombolysis in Myocardial Infarction, SAVOR-TIMI; Alogliptin after Acute Coronary Syndrome in Patients with Type 2 Diabetes, EXAMINE; y Effect of Sitagliptin on Cardiovascular
Outcomes in Type 2 Diabetes, TECOS) demostraron que saxagliptina, alogliptina y sitagliptina no estuvieron asociadas a efectos adversos cardiovasculares, mientras que respecto del desarrollo de IC saxagliptina se asoció con un 3,5\% de incidencia de hospitalización comparada con 2,8\% del placebo $(P=0,007)$, sin aumento de la mortalidad. Se esperan los resultados del estudio CAROLINA mencionado anteriormente.

En relación a los análogos del GLP-1, el estudio Liraglutide and Cardiovascular Outcomes in Type 2 Diabetes (LEADER) ${ }^{66}$ demostró con el uso de liraglutide una disminución en la tasa de mortalidad de causa cardiovascular, IAM no fatal y ACV no fatal en pacientes con DM2 con alto riesgo cardiovascular y/o IC instalada (17\% de la muestra), con menor tasa de hospitalización por IC vs placebo, siendo esta última diferencia no significativa.

- Glifozinas

El estudio Empagliflozin, Cardiovascular Outcomes, and Mortality in Type 2 Diabetes (EMPAREG) ${ }^{67}$ mostró una diferencia significativa en cuanto a reducción de la mortalidad cardiovascular por todas las causas y tasa de hospitalización por IC, sin existir diferencias en cuanto a ocurrencia de IAM y ACV. Por lo cual la última guía europea de IC la recomienda en pacientes con DM2 para prevenir o retrasar el desarrollo de IC. Por otra parte en los estudios The Canvas Program (CANVAS y CANVAS-R) ${ }^{68,69}$ el uso de canaglifozina demostró una disminución del riesgo cardiovascular (muertes de causa cardiovascular, IAM no fatal y ACV no fatal) con una diferencia significativa (que no se mantiene cuando se analiza cada variable por separado) pero con mayor riesgo de fracturas especialmente a nivel de metatarso y falanges.

\section{- Insulina}

Su utilización se relaciona con aumento de peso y retención hidrosalina, existiendo alguna preocupación respecto de la posibilidad de exacerbar un cuadro de IC basado en algunos estudios retrospectivos ${ }^{70}$. En contra partida, el estudio Diabetes Control and Complication Trial (DCCT) demostró reducción del riesgo CV mientras que el estudio Outcome Reduction with Initial Glargine Intervention (ORIGIN) no manifestó diferencias en cuanto a la tasa de hospitalización por IC. Por lo tanto, por ser uno de los fármacos claves en el tratamiento de estos pacientes y conociendo la importancia de un adecuado control metabólico, los beneficios superan los riesgos. 


\section{Discusión}

Existe evidencia considerable que el desarrollo de IC puede prevenirse y/o retrasarse mediante intervenciones destinadas a modificar hábitos del estilo de vida.

En todo paciente con DM debe evaluarse sistemáticamente la presencia de factores de riesgo cardiovascular anualmente. El tratamiento intensivo tanto de la glucemia como de los factores asociados en conjunto ha demostrado retrasar y/o prevenir el desarrollo de complicaciones macrovasculares. Esto se observa en estudios como el UKPDS, Epidemiology of Diabetes Interventions and Complications Study (EDIC) o Effect of a Multifactorial Intervention on Mortality in Type 2 Diabetes (STENO) donde el seguimiento a largo plazo mostró el impacto de la intervención temprana y del papel de la memoria glucémica. Es de destacar el impacto de las estatinas en este contexto, dado sus efectos pleiotrópicos a nivel cardiovascular al considerar a esta población plausible de tratamiento con dosis intermedias-altas.

Respecto de los fármacos empleados en los pacientes con DM, los únicos que no sólo han demostrado seguridad sino también un efecto en el plano cardiovascular son la metformina y, más recientemente, la empaglifozina y liraglutide.

En base a lo analizado se concluye que el desarrollo de enfermedad cardiovascular en la diabetes se observa como un espectro paulatino y continuo donde niveles elevados de glucemia en forma sostenida conducirán al desarrollo final de isquemia y falla cardíaca.

Nuestro rol no sólo implica un oportuno diagnóstico y un correcto tratamiento, sino fundamentalmente la educación de los pacientes para prevenir tanto el desarrollo de diabetes como de obesidad, enfermedades claves en la cascada de eventos que conducen al fallo cardíaco, lo cual se transforma finalmente en el mayor desafío.

\section{BIBLIOGRAFÍA}

1. IDF Diabetes Atlas Seventh Edition, 2015.

2. Global report on diabetes. World Health Organization, 2016.

3. Blecker S, Park H, Katz DS. Association of HbA1c with hospitalization and mortality among patients with heart failure and diabetes. BMC Cardiovascular Disorders 2016; 16:99.

4. Ryde L, Grant P, Anker S, et al. ESC Guidelines on diabetes, pre-diabetes, and cardiovascular diseases developed in coIlaboration with the EASD. European Heart Journal 2013; 34, 3035-3087.
5. Alpert A, Lavie C, Agrawal H, et al. Obesity and heart failure: epidemiology, pathophysiology, clinical manifestations, and management. Translational Research 2014; 164:1-12.

6. Etelvino GM, Peluso AA, Santos RA. New components of the renin-angiotensin system: alamandine and the mas-related $G$ protein-coupled receptor D. Curr Hypertens Rep 2014; 16:433.

7. Sarfaraz A, Jasmina V, Leanne G, et al. Angiotensin (1-12): a chymase-mediated cellular angiotensin II substrate. Curr Hypertens Rep 2014; 16: 429.

8. Mandavia C, Annauya R, Demarco V , Sower R. Molecular and metabolic mechanisms of cardiac dysfunction in diabetes. Life Sci 2013 Mar 28; 92(11):601-8

9. Morales O, Estañ Y. Conceptos nuevos sobre el sistema renina angiotensina. Hipertens Riesgo Vasc 2010; 27:211-217.

10. Mendoza-Torres E, Oyarzun A, Mondaca-Ruff D, et al. ACE2 and vasoactive peptides: novel players in cardiovascular/renal remodeling and hypertension. Ther Adv Cardiovasc Dis 2015 Aug; 9(4):217-37.

11. Márquez-Salom G. From obesity to hypertension and type 2 diabetes mellitus: the continuous metabolic multifunctional damage. Revisalud Unisucre 2013; 1: 128-130.

12. Ferrario $\mathrm{CM}$. Cardiac remodelling and RAS inhibition. Ther Adv Cardiovasc 2016; 10: 162-171.

13. Imo $E$, Brigit A, Mat JAP. Biomechanical factors as triggers of vascular growth. Cardiovascular Research 2013; 99: 276-283.

14. Grossman E, Messerli F. Hypertension and diabetes. Adv Cardiol 2008; 45:82-106.

15. Bell DS. Hypertension and diabetes: a toxic combination. Endocr Pract 2008; 14:1031-1039.

16. Sowers J, Epstein M. Diabetes, hypertension, and cardiovascular disease. An Update Hypertension 2001; 37:1053-1059.

17. Hall G. Tratado de fisiología médica. La Circulación. $12^{\mathrm{a}}$ edición. Student Consult. 2012.

18. Escudero E. Microcirculación coronaria: anatomía, fisiología y fisiopatología. Su implicancia en el estudio de la perfusión miocárdica con ecocontraste. FAC, 2001.

19. Besty B. The pathophysiology of cardiovascular disease and diabetes: beyond blood pressure and lipids. Diabetes Spectrum 2008; 21:160-165.

20. Takayuki M, SatoshiY, Hidemichi K,Tetsuji M. Diabetic cardiomyopathy: pathophysiology and clinical features. Heart Fail Rev 2013; 18: 149-166.

21. Di Carli M, Bianco-Battles D, Landa M, et al. Effects of autonomic neuropathy on coronary blood flow in patients with diabetes mellitus. Circulation 1999; 100:813-819.

22. Vinik Al, Ziegler D. Diabetic cardiovascular autonomic neuropathy. Circulation 2007; 115:387-397.

23. Gao Y, Lu B, Sun ML, et al. Comparison of atherosclerotic plaque by computed tomography angiography in patients with and without diabetes mellitus and with known or suspected coronary artery disease. Am J Cardiol 2015; 108: 809-13.

24. Low-Wang C, Hess C, Hiatt W, et al. Clinical update: cardiovascular disease in diabetes mellitus. Circulation 2016; 133:24592502.

25. Eberhard S, Michael E, Markolf H, Oliver S, Bernd S, Diethelm T. Clinical features and treatment of coronary heart disease in diabetes. John Wiley \& Sons 2015.

26. Jeffrey J, Syracuse, Chaikof E. The pathogenesis of diabetic atherosclerosis. Springer Science, 2012. 
27. Roberts K, Hevener L, Barnard J. Metabolic syndrome and insulin resistance: underlying causes and modification by exercise training. Compr Physiol 2013 Jan; 3(1):1-58.

28. Bensellam M, Laybutt $R$, Jonas $C$. The molecular mechanisms of pancreatic b-cell glucotoxicity: recent findings and future research directions. Molecular and Cellular Endocrinology 2012; 364: 1-27.

29. Philipp E, Scherer L. The multifaceted roles of adipose tissuetherapeutic targets for diabetes and beyond:The 2015 Banting Lecture. Diabetes 2016; 65:1452-1461.

30. Lam D, LeRoith D. Metabolic syndrome. En: Comprehensive free Online Endocrinology Books. South Dartmouth. MDText. com, Inc., 2000.

31. Amaral N, Darlington O. Metabolic abnormalities of the heart in type II diabetes. Diabetes \& Vascular Disease Research 2015; 12: $239-248$.

32. Thang S, Lean M. A clinical perspective of obesity, metabolic syndrome and cardiovascular disease. Journal of the Royal Society of Medicine Cardiovascular Disease 2016; 5: 1-13.

33. Halla J, Carmo J, Silva A, et al. Obesity-induced hypertension interaction of neurohumoral and renal mechanisms. Circ 2015; 116:991-1006.

34. Pasquale-Perrone F, Paolillo S, Constanzo P, et al. The role of metabolic syndrome in heart failure. European Heart Journal, 2015.

35. Ravichandran $\mathrm{R}$, Dale A, O'Shea M. Insulin resistance: metabolic mechanisms and consequences in the heart. American Heart Association 2012; 32:2068-2076.

36. Taegtmeyer $\mathrm{H}$, Beauloye $\mathrm{C}$, Harmancey $\mathrm{R}$, Hue L. Insulin resistance protects the heart from fuel overload in dysregulated metabolic states. Am J Physiol Heart Circ Physiol 2013; 305: 1693-169-7.

37. Heiko B, Dale A. Molecular mechanisms of diabetic cardiomyophaty. Diabetologia 2014; 57: 660-671.

38. Thiere J. Insuficiencia cardíaca y diabetes. Rev Argent Cardiol 2006; 74:60-67.

39. Aroor A, Chirag $\mathrm{H}$, Mandavia I, et al. Insulin resistance and heart failure: molecular mechanisms. Heart Fail Clin 2012; 8: 609-617.

40. Kok B, Brindley D. Myocardial fatty acid metabolism and lipotoxicity in the setting of insulin resistance. Heart Failure Clin 2012; 8: 643-661.

41. McMurray J, Adamopoulos S, Anker S, et al. Guía de práctica clínica de la ESC sobre diagnóstico y tratamiento de la insuficiencia cardíaca aguda y crónica 2012. Rev Esp Cardiol 2012; 65:.e1-e59.

42. Barisani L, Fernández A, Fairman E, et al. Consenso de diagnóstico y tratamiento de la insuficiencia cardíaca crónica. Rev Argent de Cardiol 2010; 78: 166-181.

43. Ponikowski P, Voors A, Anker S, et al. 2016 ESC Guidelines for the diagnosis and treatment of acute and chronic heart failure. European Heart Journal, 2016.

44. Borlaug B. Clinical manifestations and diagnosis of heart failure with preserved ejection fraction.UpToDate 2018.

45. McKelvie R, Gordon W, Ezekowitz J, et al. The 2012 Canadian Cardiovascular Society Heart Failure Management Guidelines Update: focus on acute and chronic heart failure. Canadian Journal of Cardiology 2009; 29:168-181.

46. Joso B, Neva B, et al. Silent ischemia and diabetes mellitus. Diabetologia Croatica 2010; 39-2.
47. Domanski $M$, Krause $H$, Deedwania $P$, et al. The effect of diabetes on outcomes of patients with advanced heart failure in the BEST trial. J Am Coll Cardiol 2012; 2: 914-22.

48. Gullion C, Koro C, Ephross S, et al. The incidence of congestive heart failure in type 2 diabetes an update. Diabetes Care 2004; 27:1879-1884.

49. Botta E. Insuficiencia cardíaca y diabetes: una combinación de alto riesgo. Rev Ins Car 2009; 4:107-113.

50. Lawrence $H$, Young M, Frans J. Cardiac outcomes after screening for asymptomatic coronary artery disease in patients with type 2 diabetes:The DIAD Study: a randomized controlled trial. JAMA 2009; 301: 1547-1555.

51. American Diabetes Association Standards of Medical Care in Diabetes. 2018

52. Lièvre $M$, Moulin $P$, Thivole $C$. Detection of silent myocardial ischemia in asymptomatic patients with diabetes: results of a randomized trial and meta-analysis assessing the effectiveness of systematic screening. Bio Med Central 2011; 12:23.

53. Jørgensen $P$, Jensen M, Mogelvang R, et al. Abnormal echocardiography in patients with type 2 diabetes and relation to symptoms and clinical characteristics. Diabetes \& Vascular Disease Research 2016. Reprints and permissions: sagepub. co.uk/journals Permissions.nav, 2016.

54. Sherif F, Smiseth O, Appleton C, et al. Recommendations for the evaluation of left ventricular diastolic function by echocardiography: an update from the American Society of Echocardiography and the European Association of Cardiovascular Imaging. J Am Soc Echocardiogr 2016; 29:277-314.

55. Shinder D, Kosis J, Yusuf S, et al. Diabetes mellitus, a predictor of morbidity and mortality in the studies of left ventricular dysfunction (SOLVD) trials and registry. The American Journal of Cardiology 1996; 77:1017-1020.

56. Domanski M, Krause-Steinrauf H, Deedwania $P$, et al. The effect of diabetes on outcomes of patients with advanced heart failure in the BEST trial. J Am Coll Cardiol 2003; 42:914-922.

57. McMurray J, Packer M, Desai A, et al. Angiotensin-neprilysin inhibition vs enalapril in heart failure. N Engl J Med 2014; 371:993-1004.

58. Kristensen L, Preiss D, Jhund P, et al. Risk related to pre-diabetes mellitus and diabetes mellitus in heart failure with reduced ejection fraction insights from prospective comparison of ARNI with ACEI to determine impact on global mortality and morbidity in heart failure trial. Circ Heart Fail 2016 Jan; 9(1).

59. Ferranini $E$, DeFronzo R. Impact of glucose-lowering drugs on cardiovascular disease in type 2 diabetes. European Heart Journal 2015; 36:2288-2296.

60. Tahrani A, Barnett A, Bailey C. Pharmacology and therapeutic implications of current drugs for type 2 diabetes mellitus. Nat Rev Endocrinol 2016 Oct; 12(10):566-92.

61. Luke A, Wan Z. The HbA1c and all-cause mortality relationship in patients with type 2 diabetes is J-Shaped: a meta-analysis of observational studies. Rev Diabet Stud Summer 2014; 11: 138152.

62. Eurich D, Weir D, et al. Comparative safety and effectiveness of metformin in patients with diabetes mellitus and heart failure systematic review of observational studies involving 34.000 patients. Circ Heart Fail 2013; 6:395-402.

63. Salpeter Sr, Greyber E, Pasternak GA. Risk of fatal and nonfatal lactic acidosis with metformin use in type 2 diabetes mellitus (Review). The Cochrane Collaboration 2010. 
64. MacDonald MR, Eurich DT, Majumdar SR, et al. Treatment of type 2 diabetes and outcomes in patients with heart failure: a nested case-control study from the U.K. General Practice Research Database. Diabetes Care 2010; 33:1213-1218.

65. Dormandy J, Charbonnel B, Eckland D, et al. Secondary prevention of macrovascular events in patients with type 2 diabetes in the PROactive Study (Prospective Pioglitazone Clinical Trial In Macrovascular Events): a randomised controlled trial. Lancet 2005; 366: 1279-89.

66. Marso S, Gibert D, Brown-Frandsen K, et al. Liraglutide and cardiovascular outcomes in type 2 diabetes. N Engl J Med 2016; 375:311-22.
67. Zinman B, Wanner C, Lachin J. Empagliflozin, cardiovascular outcomes, and mortality in type 2 diabetes. N Engl J Med 2015; 373: 2117-2128.

68. Bruce $\mathrm{S}$, et al. Canagliflozin and cardiovascular and renal events in type 2 diabetes. N Engl J Med 2017; 377:644-657.

69. Rådholm K, Figtree G, Perkovic V, et al. Canagliflozin and heart failure in type 2 diabetes mellitus: results from the CANVAS Program (Canagliflozin Cardiovascular Assessment Study). Circulation 2018.

70. Currie CJ, Poole CD, Evans M, et al. Mortality and other important diabetes-related outcomes with insulin vs other antihyperglycemic therapies in type 2 diabetes. J Clin Endocrinol Metab 2013; 98:668-77.

\section{Fe de erratas}

En la Revista de la Sociedad Argentina de Diabetes, Vol. 51, №3, Septiembre de 2017, pág. 96, donde figura la Tabla 2 , por un error involuntario se omitió colocar en negro las dos últimas columnas de metformina correspondientes a "30-15"

" $<15$ ó dial". La Tabla 2 correcta es la siguiente:

\begin{tabular}{|c|c|c|c|c|c|}
\hline & \multicolumn{4}{|c|}{ Clearance de creatinina $(\mathrm{ml} / \mathrm{min})$} & \multirow[b]{2}{*}{$<15$ ó dial } \\
\hline & $\geq 60$ & $60-45$ & $45-30$ & $30-15$ & \\
\hline \multicolumn{6}{|l|}{ Metformina } \\
\hline Sulfonilureas & & & & $\begin{array}{l}\text { Glipizida y } \\
\text { gliclazida }\end{array}$ & \\
\hline \multicolumn{6}{|l|}{ Repaglinida } \\
\hline \multicolumn{6}{|l|}{ Pioglitazona } \\
\hline \multicolumn{6}{|l|}{ GLP-1 ag } \\
\hline \multicolumn{6}{|l|}{ (-) DPP-4 } \\
\hline (-) SGLT-2 & & $\begin{array}{l}\text { Canagliflozina } \\
\text { y empagliflozina }\end{array}$ & & & \\
\hline Insulina & & & & & \\
\hline
\end{tabular}

Tabla 2: El color blanco representa que la indicación del fármaco no merece mayor cuidado con respecto al filtrado; el color gris oscuro significa que debe tenerse precaución, ya sea por ser drogas capaces de producir hipoglucemias (como es el caso de la glipizida, gliclazida y repaglinida) o por generar retención hidrosalina y edemas (pioglitazona), etc.; el color negro indica que la droga o familia de drogas se encuentra contraindicada en el filtrado glomerular señalado. Adaptado de las normas KDOOI. 